\title{
La santé mobile nous rattrape - avec quelles conséquences?
}

\section{Yvonne Gilli}

Dr méd., membre du Comité central de la FMH, responsable du département Numérisation / eHealth

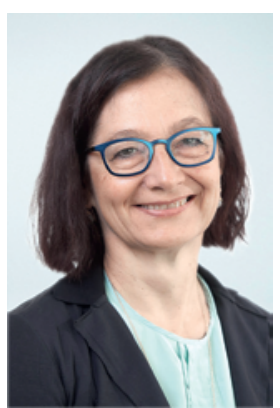

La santé mobile désigne les applications en lien avec la santé sur les appareils mobiles comme les smartphones, les tablettes, les montres connectées, etc. Elle inclut également les informations médicales collectées grâce à des capteurs. La santé mobile recouvre donc tout un pan de la cybersanté (eHealth) qui, elle, fédère tous les services électroniques en lien avec la santé [1]. Pour certains experts, l'évolution et la diffusion fulgurantes de la santé mobile marquent une rupture à l'instar d'internet, qui a modifié en profondeur notre rapport à l'information[2]. La frénésie avec laquelle la santé mobile se positionne sur le marché principalement grâce au

\section{Les compétences numériques requièrent} une formation professionnelle du corps médical pendant la formation pré- et postgraduée.

développement technologique est énorme. En 2014, pas moins de 6 milliards d'abonnements mobiles étaient souscrits dans le monde [3]. Aujourd'hui, nous estimons qu'entre 200000 et 300000 applications sont présentes sur le marché, et que 500 millions de personnes les utilisent, en grande partie indépendamment du corps médical [4]. Il s'agit là du secteur industriel qui connaît la plus forte croissance à l'échelle mondiale.

1 http://www.e-healthsuisse.ch/grundlagen/ index.html?lang=fr

2 http://www.hkstrategies. be/en/Insights/What-isnext-for-mHealth-inEurope\#.WMWtU2_hDIU 3 Prof. Christoph Thuemmler, IoT Week 2015,

Lisbonne. Présentation «IoT Analytics for smart Health and Care».

4 https://www.publicpolicy exchange.co.uk/events/ HB28-PPE2. Présentation Dee O'Sullivan, Managing Director, myhealthapps. net at PatientView

5 Prof. Christoph Tuemmler, Professor of Health, Edin burgh Napier University $6 \mathrm{http} / / / \mathrm{www} \cdot h$ healthpar

liament.eu/recommenda tions-2016
Il est évident que cette évolution n'est pas sans conséquence sur le domaine de la santé. Lorsque les technologies se développent rapidement, comme de la communication, les acteurs concernés sont généralement dépassés. Moteurs de cette évolution, les intérêts économiques particuliers représentent un véritable défi éthique dans un marché aussi sensible et réglementé que celui de la santé. Opportunités et risques doivent être traduits en hypothèses et examinés à l'aune des valeurs déontologiques fondamentales et de l'analyse des tendances. En février de cette année, Public Policy Exchange a organisé à Bruxelles un congrès pour les professionnels de santé intitulé «The future of Mobile Health in Europe».

A cette occasion, le Prof. Thuemmler [5] a parfaitement résumé ces tendances: c'est le cas pour les technologies de l'information et
- Toujours plus de données sont collectées et traitées sur les appareils mobiles, aussi bien par les patients que par les médecins.

- Selon l'application, les données sont traitées et collectées à des endroits différents: localement (Edge Computing) ou sur internet (Cloud Computing)

- Le traitement des données suit toujours plus les mécanismes neurobiologiques (Neuroempiricism).

- Les intérêts industriels des fournisseurs internationaux et nationaux (Apple, Google, Microsoft, Sanofi, Siemens, Swisscom, etc.) comportent des risques en termes de protection et de sécurité des données.

Pour les médecins, la question qui se pose est de savoir comment acquérir les aptitudes nécessaires et organiser leur environnement de travail à l'ère 4.0. En 2016, un groupe de jeunes professionnels de la santé a publié une enquête sur les compétences numériques des médecins européens[6]. Plus de la moitié a déclaré n'avoir reçu aucune formation professionnelle dans ce domaine. Deux recommandations sont ressorties de cette enquête:

- Les compétences numériques doivent être intégrées de manière adaptée et obligatoire dans la formation.

- Le recours aux compétences numériques peut être soutenu par des systèmes d'indemnisation adaptés.

A l'ère de la santé mobile et de la cybersanté, l'activité médicale reste une prestation d'humain à humain.

Pour les médecins suisses, ces recommandations pragmatiques impliquent la création d'un cursus postgrade en informatique médicale. Mais n'oublions pas qu'à l'ère du numérique aussi, l'activité médicale reste une prestation d'humain à humain. Les moyens alloués à la numérisation, la cybersanté et la santé mobile seront toujours en concurrence avec ceux alloués à l'enseignement des valeurs fondamentales de la médecine telles que les compétences sociales, la réflexion sur soi et l'éthique clinique. 